\title{
Téoros
}

Revue de recherche en tourisme

\section{L'accueil touristique vu comme comportement de communication}

\section{Marc Laplante}

Volume 10, numéro 2, juillet 1991

Accueil et tourisme

URI : https://id.erudit.org/iderudit/1078957ar

DOI : https://doi.org/10.7202/1078957ar

Aller au sommaire du numéro

Éditeur(s)

Université du Québec à Montréal

ISSN

0712-8657 (imprimé)

1923-2705 (numérique)

Découvrir la revue

Citer cet article

Laplante, M. (1991). L'accueil touristique vu comme comportement de communication. Téoros, 10(2), 20-23. https://doi.org/10.7202/1078957ar d'utilisation que vous pouvez consulter en ligne.

https://apropos.erudit.org/fr/usagers/politique-dutilisation/ 
L'hospitalité touristique: une communication particulière

À première vue, l'hospitalité est essentiellement communication. Mais l'hospitalité touristique est une communication qui a ses caractéristiques propres puisqu'elles'établit entre des interlocuteurs particuliers: les visiteurs-touristes et les visités-hốtes. Cette spécificité tient au fait que les gestes d'accueil de l'hôte sont partie intégrante de l'expérience du visiteur: dans le contrat de service qui lie le touriste à sa destination, il y $\mathrm{a}_{\text {, }}$ explicitement et implicitement, l'accueil. Explicitement car le touriste s'attend à ce qu"on le serve car il paie pour cela (services compris!); implicitement parce qu'au-delà de l'hospitalité assurée par les personnes de la profession, il espere qu'on l'acceptera et qu'il se sentira le bienvenu dans la société où il a choisi de passer ses vacances ${ }^{(1)}$. Ce eon\%, dans ce cas, réfere à la population en général et aux personnels des services non spécifiquementtouristiques que le touriste utilisera au cours de son séjour.

\section{Les relations touristiques visiteurs-} visités: une communication dite difficile

On a déjà beaucoup écrit sur les relations touristiques entre visiteurs et visités; on a surtout décrit comment elles étaient difficiles $^{(2)}$. Lesdeux interlocuteursen présence sont dans des contes très différents (ex.: vacances-travail); l'un est un étranger de passage et l'autre est un résident; les conditions de travail et de vie des personnels qui servent les touristes sont souvent peu gratifiantes, etc.

Sous ces nombreux et divers écrits, il faut chercher surtout ce qui reste constant; il nous semble que plusieurs de ces difficultes de communication entre les visiteurs et les visités tiennent à quelques traits propres au statut de touriste: celui-ci est, par définition, un étranger en vacances. Son statut d'étranger ne devrait pas poserde problèmes car, de tout temps, l'hospitalité s'est exercée particulièrement envers l'étranger ${ }^{(3)}$. Mais l'étranger, objet de l'assistance de l'hôte,était plutốt le pèlerin au sens large du terme, plus large que celui de voyageur accomplissant un rituel religieux. Son vo-

Monsieur Marc Laplante est professeur au Departement d'ettudes urbaines et touristiques de PUniversité du Québec á Montréal. yage était une sorte de quête de savoir, une exploration dans l'inconnu, ou bien une mission pour créer des liens et commencer des échanges. Voyager comportait également bien des dangers et 1'aide à un voyageur contenait une idée de réciprocité, l'idée d'être aidé à son tour si on voyageait. Bref, ce voyageur n"était pas touriste.

Il faut donc revenir au statut de vacancier pour tenter d'expliquer - en partic - certains problemes des relations entre touristes et visités. Il s'agit, curieusement, d'un aspect de l'expérience touristique dont on parle peu. Pourtant, on peut, sans forcer les faits, interpréter cette expérience à partir de ce qu'on connait, en sciences sociales, du jeu et de la fêtete ${ }^{(4)}$. Pour dire les choses en quelques mots, l'esprit ludique et festif qui marque la totalité du voyage d'agrément induit des comportements et des attitudes typiques qui peuvent être adérangeantes» pour les gens qui entourent les touristes et qui ne sont pas dans cet esprit: tendances à $l^{\prime}$ exubérance, à la transgression de certains codes et règles habituellement respectés, sentiment d'ettre dans «autre monde» ayant son espace-temps propre ${ }^{(5)}$ avec, pour conséquence, une certaine absence des réalités du lieu visité (son histoire, ses problèmes politiques, etc.) et surtout, une motivation dominante, celle de la recherche du plaisir, de l'agrément justementetconséquemment, une capacité d'abandonner rapidement tout ce qui pourrait être ennuyeux, sérieux, difficile, etc.

Si on s'en donnait la peine, on pourrait, par simple observation rigoureuse des comportements touristiques les plus typiques (et souvent les plus décriés), vérifier combien plusieurs critiques négatives à propos des touristes sont inspirées de cette méconnaissance des dimensions ludiques de l'expérience touristique.

Le voyage de vacances, enfin, appartient au champ plus vaste des activités de temps libre et de loisir. Sociologiquement, ce temps libre n'a de sens que par rapport aux autres temps sociaux, ceux de la vie professionnelle, de la famille, de l'engagement social, etc ${ }^{(6)}$. Parmi les enseignements de cette sociologie du loisir, il nous importe de retenir pour notre propos que le temps libre est celui des relations sociales primaires (empathiques, face-à-face, entre amis, parents, copains de travail, etc.) par opposition notamment au temps de travail dominé par des relations sociales secondaires (froides, hiérarchiques, anonymes, etc.). Les expériences de temps libre se vivent donc souvent dans l'intimité des couples, des groupes d'amis ou de parents, des voisins et compagnons, etc. Ceci constitue une sorte de bulle sociale dans laquelle chacun se sent bien, accepté, valorisé. Quand vient le départ de vacances, la grande majoritê des gens amènentavec eux cette bulle sociale et les expériences vécues à l'étranger seront d'abord partagées par les membres de ces bulles sociales. On comprendra alors que ces petits uclans s"autosuffisent socialement, ce qui ne favorise pas la recherche des contacts nouveaux dans le milieu visitép.

Le temps du voyage d'agrément n'a donc que peu de ressemblance avec celui du voyage d'affaires ou d'études. C'est un temps dont on dispose à son gré, pour faire ce qui nous plaît, un temps moins réglé, moins normé, plus propice aux expériences nouvelles, aux essais de toutes sortes «juste pour jouers, et donc, finalement, un temps souvent créateur parce que moins contraint. par les devoirs, les routines et les échéances.

Si on veut bien se rappeler, en résumé, que le touriste, peu importe ses autres deffinitions, est un étranger, en vacances, à la recherche de changement, on sera moins étonné du contexte tout à fait particulier dans lequel se trouveront les touristes et leurs hôtes pour communiquer. Et les exigences de l'accueil de ces touristes deviendront plus évidentes.

\section{Un petit détour par les sciences de la communication}

Il faudrait, pour bien faire, introduire une longue parenthèse pour dire comment les diverses disciplines spécialisées qui forment les sciences de la communication pourraient contribuer à l'analyse et à la compréhension de ce qui se passe entre les touristes et les visités. Mais ceci nous éloignerait trop du principal objectif du présent texte: sensibiliser les intervenants touristiques au fait que l'hospitalitế n'est pas qu'une affaire de bonnes dispositions envers autrui et conséquemment que l'expérience pratique, seule, est insuffisante pour garantir un accueil touristique de grande qualité.

Pour atteindre cet objectif, nous pouvons simplement exploiter les acquis les plus 
assurés des travaux de recherche en communication. Dans ce domaine, de grandes decouvertes ont ếté faites au cours des 30 40 dernières années. Un livre, entre autre, a fait un bilan à ce propos, celui de Paul Watzlavick, Janet $\mathrm{H}$. Beavin et Don D. Jackson: Une logique de la communication ${ }^{(8)}$. Cedocumentétait désigné, en 1981, comme un «classique $w_{4}$ un textede référence pour comprendre la «nouvelle communications ${ }^{\prime 9}$. Il commence par un énoncé de certains axiomes $^{(10)}$ sur lesquels se sont fondés les nouvelles recherches. Sur de telles prómisses, nous pouvons, à notre tour, nous appuyer pour enclencher diverses réflexions sur les conmunications entre les touristes et les visités ${ }^{(11)}$.

\section{Tout comportement est communication}

aLes membres d'une société donnée partagent des milliers de types de comportements eprogrammés par l'appartenance à une culture, sous-culture ou tradition familiale déterminées. Certains de ces comportements peuvent ne pas avoir les mêmes connotations pour un étranger. Les ethnologues nous apprennent qu'il existe littéralement selon les cultures des centaines de façons, par exemple, de saluer ou d'exprimer la joie ou la douleur. C'est l'une des lois fondamentales de la communication que tout comportementen présence d'autrui a valeur de message, en ce sens qu'il definit et modifie le rapport entre les personness ${ }^{(12)}$.

C'est le premier des axiomes de la communication: tout comportement a valeur de message en présence d'autrui. Et un comportement ne peut pas avoir de contraire: le non-comportement n'existe pas. Donc, on ne peut pas ne pas communiquer: les silences sont éloquents; les refus de communiquer sont aussi des messages.

Le touriste et ses hôtes sont alors constamment en train de communiquer quand ils sont en présence l'un de l'autre. Enfermé dans sa chambre d'hôtel, le touriste envoie encore des messages aux personnels d'entretien. Le touriste qui ne parlerait pas de tout son voyage parlerait quand meme par ses gestes, ses mimiques, ses facons de s'habiller, de se comporter, de se présenter, etc. Et tous ces messages vont influencer les comportementsde ceux qui les recoivent.

La toute première conséquence de cet axiome est qu'il importe d'etudier tout ce qui se passe entre les touristes et les visités. Il ne suffit pas, par exemple, d'informer les gens de $1^{f}$ accueil sur les motivations et les attentes générales des touristes en matière d'hospitalité; il ne suffit pas non plus d'inculquer à ces personnels les règles de base de la politesse ou de la courtoisie. Quiconque fait de l'accueil vit en présence des touristes et doit avoir conscience des processus dans lesquels il s'engage dès le premier contact. Car il s'agit bien de processus de communication, comme le rappelle le second axiome.

\section{La communication est un processus stochastique}

Ce qui se passe entre deux personnes (ou deux groupes, ou deux pays) en présence l'une de l'autre n'est pas tout à fait aleatoire. En communiquant de plus en plus, ces deux personnes acquièrent, sans lesavoir consciemment, des règles, des habitudes; elles apprennent et, d'une fois à l'autre, elles $n$ ont pas à tout recommencer; elles stockent les adaptations antérieures pour s'en servir ultérieurement.

Ce processus se déroule, il faut bien le noter, sans que les interlocuteurs starrêtent pour prendre conscience de ce qui se passe entre eux. Pour comprendre de tels processus, il faut être un observateur extérieur, se placer hors de l'interaction elle-même et il faut arriver à parler sur la communication qui se passe sous nos yeux (ce qu'on désigne comme méta-communication).

Pour l'observateur, du point de vue de la méta-communication, toute interaction apparaît, par analogie, comme un jeu, c'est-àdire, un ensemble de scoups régis par des règles précises. Connaissantces règles, ces successions de acoupss (comme au jeu $\mathrm{d}^{\prime} \mathrm{echec}^{\prime}$, on peut asuivres $\mathrm{l}^{\prime}$ évolution $\mathrm{d}^{\prime}$ une interaction. Dans une séquence de communication, tout ếchange de message restreint le nombre d'échanges suivants possibles (comme aux bchecs, tout coup restreint le nombre des coups suivants possibles). Les messages ouvertement échangés deviennent partie intégrante du contexte interpersonnel et marquent les limites d'une interaction ultérieure.

Il y aurait beaucoup à dire sur les communications entre touristes et visités à partir de cet axiome. Mais il faut parler au conditionnel puisque nous ne disposons pratiquement pas de données pour faire de la méta-communication à ce propos, c'est-àdire pour parler de l'evolution des interactions entre touristes et visites. Que savonsnous de l'evolution des rapports entre un guide touristique, par exemple, et le groupe dont il a charge pour trois heures, une joumée ou une semaine?(13) Comment changent les interactions entre un touriste et les divers personnels d'un hôtel selon que le touriste y reste un jour, trois ou dix jours? Qui a jamais étudié l'histoire d'une expérience vécue par un touriste quantà ses rencontres avec des gens du pays, hors des services touristiques?

D'autre part, même si ces connaissances étaient acquises, elles ne toucheraient qu' une partie des interactions totales $\mathrm{d}^{7} \mathrm{un}$ touriste avec le milieu qu'il visite. Plusieurs de ces interactions seront difficiles à évaluer parce qu'elles sont souvent brèves ou même très brèves, parce qu'elles consistent souvent en demandes d'information ou d'aide, parce que, du côté des visités, plusieurs communications sont mécanises et à sens unique vers les touristes (ex: un centred interprétation ou un musée avec un système pré-enregistré de messager pour guider les visiteurs et les sensibiliser aux choses à voir), etc.

Malgré ces difficultés et 1 absence de recherche, il faut garder à l'esprit que le touriste reçoit et émet, d'heure en heure durant tout son voyage, des messages qui influencent son experience et compte beaucoup dans le bilan final qu'il fera de celleci. A mesure qu'il communique, le touriste accumule desexpériences d'interaction; des modèles s'élaborent: par exemple, il se sentira de plusenplusa $l^{\prime}$ aise pours'engager dans des échanges plus personnels ou bien de plus en plus gêné de parler de lui, de son pays, des découvertes qu il a faites ici, etc. Pourquoi certains touristes ne parlent-ils que de la pluie ou du beau temps avec ceux et celles qu'ils rencontrent? Parce qu'ils n'ont rien d'autre à dire? Reponse trop facile! Réponse peut-être qui exprime un refus de communiquer avec les touristes.

Même en ne faisant qu'effleurer ces questions, on peut imaginer comment leur approfondissement viendrait changer tous nos entendements et nos idées acquises sur l'accueil touristique. Etces nouvelles compréhensions pourraient changer bien des choses. Pensons, par exemple, que l'apprentissage des interactions, acquis par un touriste avec le Québec et les gens qui l'ont reçu, sera transmise à d'autres qui viendront ici, le bouche-à-oreille étant la plus efficace des publicités; pensons aussi qu'une expérience acquise à ce propos a une destination donné risque de servir lors d'un voyage vers une autre destination et que les comparaisons ne manqueront pas de S'établir entre divers lieux de vacances touristiques quantà la qualité des échanges entre visites et touristes.

Profitons de cet axiome et des quelques réflexions qu'il a suscité pour interroger une des images fortesdu produit touristique québécois: la chaleur de l'accueil ( $\alpha \mathrm{It}^{7} \mathrm{~s}$ warmer up Norths, theme qui joue sur le paradoxe puisque le Nord est associé au froid; or la communication paradoxale est dangereuse et la psychiatrie en sait quelque chose puisqu'on la retrouve chez le schizophrène1). La joie de vivre, la porte ouverte, la porte d'entrée de l'Amérique, le sens de la fête, etc., sont autant de sous-thèmes qui n'envoient qu' un message: au Québec, on est content de vous recevoir, on aime la visite. Ceci est fort sympathique mais fixe aussi très haute la barre de qualité des gestes $\mathrm{d}^{7}$ accueil et d'hospitalité. A promettre autant, il fautdonner beaucoup. Nous aimons beaucoup, c'est un fait, recevoir des 
parents et des amis et fêter avec eux et ce fait s'explique historiquement; mais en estil également ainsi envers les étrangers? Et notamment envers ces étrangers de passage que sont les touristes? ${ }^{24)}$ Les Américains viennent-ils moins au Québec simplement parce que nous ne sommes plus une société aussi exotique qu'avant depuis notre modernisation? Comment étaient-ils reçus ici dans les années 1920,1930 ou 1940 ? Qu'ont-ils accumulé comme expériences d'interactions avec le Québec depuis les années 1960 , la révolution tranquille - et moins tranquille - les crises politiques?

A partir donc de petites questions sur les processus d'interaction entre touristes et visités, on pourrait traiter de problèmes nettement plus complexes et conséquents pour $1^{\dagger}$ avenir dı tourisme québécois.

Une communication se fait toujours simultanément à deux niveaux: celui du contenu et celui de la relation

Le niveau du contenu transmet de l'information et celui de la relation induit un comportement.

Il est rare que les relations soient explicitement définies. En général, quand ça vā bien, on echange et on ne s'arréte pas à la relation. Mais quand 1 'interaction va mal, le débat porte de plus en plus sur la relation et le contenu perd de son importance.

Il s'agit ici d'un axiome très conséquent pour comprendre comment se dêroule une interaction. Si les partenaires sentent qu'ils s'entendent et que les messages émis sont bien reçus, ils ne s'interrogeront pas sur la relation qu'ils sont en train de vivre; sinon, ils vont introduire dans cette relation des interrogations surcelle-ci même. Quelqu'un demandant à son vis-ă-vis: «Tu me comprends? $*$ ou précisant: «Ne t'inquiètespas, je plaisantais», communique sur sa relation avec l'autre. Si les choses se compliquent, l'un dira à l'autre: «Tu sembles toujours dans la lune quand je te parle! $\%$; l'autre réagira: a Ta manière de dire les choses me dérange; j"aime mieux ne rien entendre! $\$$. En psycho-sociologie des relations conjugales, on a connu des couples qui avaient vécus une dizaine d'années à nẹ parler que de leurs relations, sans se souvenir des contenus. Les bchanges avaient pris la forme $d^{+}$une esuite infinie alternée $*$, comme on dit en mathématique! Le défi, pour le clinicien, fut d'interrompre cette suite: aussisimple que de briser un cercle vicieux... Pratiquement, retenons que toute insistance pour parler, décrire, expliquer les relations entre $A$ et $B$ peutservir d'indice pour déduire que ces relations font problème. Prenons l'exemple des relations Québec-Canada, sans insister! Obnubilés par les difficultés de leurs rapports dont les échos remplissent nos mass média depuis 10-20 ans, les leaders de part et d'autre n'ont mẹme pas pu échanger sur un contenu aussi mince que celui des propositions du Lac Meech!

En tourisme, qu'en est-il? Nous pourrions dire que l'insistance à s'occuper de la concurrence, à devenir compétitif, de même que la primauté accordéce à la fabrication des images de nos produits sont des actes qui trahissent davantage nos inquiétudes quant à nos relations avec le tourisme que nos intérêts à livrer du contenu. Il y a $30-40$ ans, le Québec n'hésitait pas à produire luimême des guides touristiques de $300-400$ pages, racontant son histoire, son mode de vie, ses particularités locales et régionales, etc. Les messages de ces guides dépassaient de beaucoup la stricte transmission d'information pour exprimer un accord profond entre l'émetteur et ce qu'il voulait dire. De plus en plus, on ne sait plus comment dire le Québec ${ }^{(15)_{+}}$des slogans vaporeux comme aC'est beau, c'est grand. c'est à voirs, que la Chine, la Russie, la Sibérie même auraient pu utiliser mieux que nous, une quinzaine de guides régionaux (qui, réunis, donneraient un guide de \pm 1500 pages pour le Québec!) riches en informations techniques mais très répétitifs par leur forme et souvent, par leurs contenus (toujours de la nature et de la culture, de l'histoire et de l'actuel, des événements en quantité, etc.). Peut-être utiles aux touristes une fois rendus dans une région, ces documents - les plus officiels parce que produits avec le ministère du Tourisme disent surtout nos difficultés actuelles d'identité. Elles sous-entendent que nous sommes plus préoccupés des relations entre nos régions que d'échanges avec les futurs touristes sur ce qui fait du Québec une destination unique.

Ne faudrait-il pas considérer enfin que la production même du présent numéro de Téoros sur l'accueil est un indice d'une préoccupation dominante pour les relations avec les touristes? Son thème ne s'appuiet-il pas sur une constante inquiétude depuis quelques années pour une meilleure qualité des services touristiques? Cette qualité, qu' on veut atteindre par plus de profession. nalisme n' implique-t-elle pas, notamment, a une conscience plus grande de l'acte d'accueil lui-même?

\section{L'homme seul, utilise deux modes de communication: le digital et l'analogique}

La communication digitale est plus logique et plus précise, plus abstraite etrationnelle; elle transmet des savoirs. Elle est plus récente dans $1^{*}$ histoire humaine et sert surtout à nommer les choses. La communication analogique réfere à tous ce qui est transmis en plus du nom des choses, de l' information: le non-verbal, les gestes, les sentiments et les émotions qui ne se disent pas clairement avec des mots, etc.
En rapport avec l'axiome précédent, l'échange des informations est une communication davantage digitale alors que l'échange sur les relations est obligée de se faire le plus souvent sur le mode analogique.

L'homme, en tant qu'émetteur comme en tant que récepteur, doit constamment combiner ces deux modes et traduire l'un dans l'autre. Le passage du digitalà l'analogique entraine une perte d'information (ex. monter une pièce de thếtre comme l'Histoire d'un Royaume, au Saguenay, pour faire connaître l'histoire régionale); le passage de l'analogique au digital pose le probleme de dire avec des mots des choses difficiles à formuler (ex.: le contrat de mariage, communication sur le mode digital, traduit mal $1^{t}$ amour entre deux êtres, communication sur le mode analogique).

L'accueil, l'hospitalité, appartiennent primordialement au monde de la communication analogique. On veut dire à l'autre - lo touriste - qu' on l'aime bien, qu'on le désire, qu'il est attendu, accepté. Comment le dire? «Dites bonjour» est une très faible expression digitale pour aider les gens de l'accueil à exprimer les sentiments d'un hôte véritable. L'auto-collant «Bonjour», apposé à toutes les portes des lieux de services touristiques est d'un laconisme extrême. Comment ce mot, collé sur la porte, est-il en rapport avec ce qui se passera derrière cette porte? Remplace-t-il un sbonjours de vive voix qui dit toujours plus que le mot? Prépare-t-il à un abonjours plus chaud à l'intérieur? Que penser d'affiches illustrant 1'hospitalité des Québécois (communication analogique) apposées dans un bureau d'information touristique ou on est lent à répondre, ou le préposé fait son «job» comme un robot, ou on a multiplié les présentoirs et les machines pour l'autoinformation? Ne s'agit-il pas de communications digitales pauvres pour traduire les messages analogiques des affiches sur les murs?

Dans une longue séquence d'échanges, émetteurs et récepteurs ponctuent, de fait, la séquence de manière que l'un des deux semblera avoir l'initiative ou la prédominance, ou le statut de dépendance, etc.

Selon sa culture, sa position dans la société, chacun partageavec les autres beaucoup de conventions (implicites) de ponctuation de la séquence des faits. Par exemple, on accepte qu'une personne reconnue par d'autres comme un leader d'opinions dans un domaine s'exprime en premier sur un sujet donné dans ce domaine.

On retiendra surtout de cet axiome que beaucoup de conflits sur les relations viennent de désaccords sur la manière de ponctuer laséquence des faits. Un exemple d'un cas typique: une discussion à propos d'une 
dispute entre deux voisins ou deux associés pour établir qui a commencé!

Ce sujet est de première importance car il réfere à la manière dont se structure une interaction.

A propos des relations touristiques, cet axiome pose une question stratégique: qui a commencet la relation? Nous avons souvent pris au piège des étudiants et des intervenants en tourisme avec cette question. On nous a gếnéralement répondu quẹ le touriste commençait l'interaction en débarquant chez nous... Mais ce touriste n'a-t-il pas ếté invité par nous? Combien dépensons-nous par année pour l'inviter, le solliciter, le prier même de venir nous voir? Nous sommes totalement ouverts à ces touristes et nous le faisons savoir partout (quand 1'Albanie était totalement fermée, les touristes n"y allaient pas).

Si on dit à quelqu'un: Venez me voirs, quand il arrive chez nous, il répond à cette invitation. Le Québec a donc commencé I'interaction par une action d'invitation; l'arrivée du touriste iciest la réaction à cette action. Pour que l'interaction commence vraiment, c'est à nous en tant qu'hôte, de poser le troisième geste: l'accueillir rócllement. Si nous oublions cette séquence, si nous persistons à penser que le premier pas a été fait par le touriste quand il est entré chez nous, il y a de fortes chances qu'une mésentente - implicite mais ressentie $s$ installe entre les deux parties. Qui considère que le touriste dérange, soit par son trop grand nombre, soit par ses comportements, agit comme s'il ne l'avait pas invité (ou comme s'il avait invitê sans savoir qui il invitait). Le touriste, dûment invité, mais ne trouvant qu'indifférence ou morosité autour de lui, se sentira comme un intrus dans une soirée où il n'a pas été invité. Geste apres geste d"accueil durant tout son séjour peuvent rester marqués par ce malaise au début de l'interaction.

Une grande partie de ce probleme typique de l'accueil tient probablement au fait que l'invitation adressée à la planète entière passe pour être faite au nom de tout le Québec, et donc de tous les Québécois, puisque le gouvernement n'est activement associé à ces actes d'invitation. Mais en fait, la très vaste majorité des résidents pensent que cette invitation ne sert que ceux qui vivent du tourisme. L incessant message de l'industrie et du gouvemement pour faire savoir au Québécois combien le tourisme est important, combien il crée d'emplois, comment il est ou devient notre 3e, 2 e ou lère industrie, etc. A-t-il d'autres sens que d'essayer de dire à tous qu'ils doivent contribuer à l'accueil? Et que dire d'une opération comme la décoration de la famille hospitalière de l'année? D'une cérémonie comme l'attribution des Grands prix du tourisme (donne-t-on des grands prix de la religion, de la famille, du syndicalisme, du loisir? On peuty voir autant de tentatives pour que l'ensemble de la population prolonge les efforts des intervenants touristiques afin d'assurer le meilleur accueil possible. II y a peu à redire de ces initiatives sauf à en questionner l'efficacité. Car, aujourd'hui plus qu'hier encore, on semble vouloir davantage les retombees économiques du tourisme que les touristes eux-mêmes! Et ceci n'échappe pas au commun des mortels qui, de villes en villages, voient et entendent les intervenants touristiquespresqu'exclusivement occupés de ces mirifiques retombées. Comment convaincre tout un chacun jusqu'au fond des campagnes qu'il a invité les touristes, qu'il y va de ses intéréts qu'ils viennent nombreux, séjournent longtemps et circulent partout?

La prochaine mission des promoteurs du tourisme ne serait-elle pas de travailler à réduire les désaccords sur la ponctuation de la séquence des interactions entre les touristes et les visités?

\section{Conclusion}

Le libellế de la dernière question semblera du charabia pour qui n'aura pas lu les pages qui précedent; pour les autres, il sera clair que toute question relative aux échanges entre visiteurs et visités nous amène à nous situer au niveau d'une méta-communication. Or, al'aptitude à méta-communiquer de façon satisfaisante n'est pas seulement la condition sine qua non d"une bonne communication, ellea aussi des liens très étroits avec le vaste problème de la conscience de soi et d'autruis, (16)

II nous est arrivé fréquemment dans le passé de souhaiter qu'un maximum d'efforts soit donné à la connaissance approfondie du touriste; nous avons aussi souvent insistésur la nécessitéd'analyser l'expérience touristique comme un tout et non pas en pièces détachées. L'accueil touristique, tel qu'abordé ici, apparaît comme faisant partie de cette experience et mème comme un possible fil d'Ariane réunissant toutes les expériences touristiques particulières. Et cetaccueil, il nous appartienten tantqu'hồte de l'assurer. A la lumière des sciences de la communication - lumiere beaucoup plus éclairante que le contenu du présent texte peut le suggérer - il faudra repenser largement l'action des hôtes vis-à-vis ce touriste encoremal connu, cetétranger, en vacances, a la recherche de changements qui communique constamment avec nous et avec qui il nous faut obligatoirement communiquer constamment.

\section{Notes explicatives}

(1) Conformement a l'approche sociologique, nous réservons le mot touriste pour dessigner celui ou celle qui fait un voyage d'agrément. un voyage donc durant ses temps libres de week-end ou de vacances.

(2) THUROT, $J$-M, et autres (1976) ont essaver de faire un bilan des difficultes de la rencontre du touriste et de son hôte parce que cette rencontre était caractérisee par: son caractere trans toire. des contraintes temporelles et spatiales, des rela tions inegales et une absence de gratuité initiale des rapports. Voir: Les effets du tourisme sur les valeurs socio-culturelles, Aix-en-Prowence, C.H.E.T, strie C, no 34, pp. 15 et suivanteg. $\mathrm{Ce}$ bilan conceme davantace les relations entre les touristes et les pays en vole de développement. Pour une vue d'ensemble, on consultera: SEY. DOUX, Jose (1983), De l'hospitalitd â l'accueil Spes, Delta. WAKERMANN, Grabriel (1988) traite aussi de ce contexte des rencontres dans Le tourisme international. Pans, A. Collin.

(3) Voir, di ce propos, le beau texte de I'histonen Fustel de Coulange, N. D : La cilte antique (Paris, Hachette, 19521 qui fappelle lionigno des rituels de l'hospitalité à l'aube des civilisations.

(4) Voir a ce propos: LAPLANTE, Marc (1969), kLta révolution du wovage d’agréments, in Lolsir et Sociate, wol. 11, no 1, pp, 11-32. On consultera Également Táoros; wol, 5, no 2, juillet 1905, consacre aux thtes ot bionements.

(5) JAFARI, Jafar (1988), dans le numéro de Táoros cite ci-haut (pp. 59-80) presente un modile qui Mlustre tres precisement les changements qu s'operent quand un individu passe de son monde ordinaire quotidien au monde non-ordinalre de son temps de voyage de vacanoes.

(6) PRONOVOST, Gilles (1984), Temps, loisir at soclèté, Sillery, P.U.O

(7) Uns des constantes de l'observation systemique de longue duree de petits groupes touristiques est l'interaction continue entre les membres d'un même wparty a à tous les moments d'une visite de ville, par exemple. Notes de terrain; recharches exploratoires sur les interactions visiteurs visites Qubbec durant l'été 1986

(B) Paris, Seuil, collection a Pointso, 1979, chapitre 2 pp. 46-69: a Propositions pour uné axiomatique de la comminication. Le lure onginal en langue anglaise est paruen 1967: Pragmatics of Human Communleation, New York, Noton.

(9) Titre d'une publication produite en 1981 (Paris, Seuil, collection a Points * $)$, regroupant des texters revelateurs de diverses ecoles de recherche aux Etats-Unis, dominées par des auteurs reputes: comme Bateson, Birdwhistell, Goffman. Hall, Jackson, Scheflen, Sigman, Watzlavick, etc. Ces textes furent recueillis et présentés par Yves Winkin.

(10) Prémisseconsidérécocomme évidente, et recue pour vraie sans demonstration par tous ceux oul en comprennent le sensis. LALANDE, André (1972). Vocabulaire technique et critique de la philosophie. Paris, PU.F. D. 105: les sens A celui cité ici, est consideré par l'auteur comme le plus visuel.

(11) Les axiomes, comme l'ensemble du livre qui sert ici de reference, scrit particuliefement utiles a l'étude des interactions inter-individuelles; il faut proceder avec prudence quand on cherche a les utiliser, comme nous le ferons ici, pour discuter de relationsqui ne sont pas uniquement interperson: nelles, mais aussi, intergroupes et interculturel. les.

(12) WATLLAVICK, Paul(1976), La réalité de la réalité, Paris, Seul, p. 16

(13) On a dejât tudie du point de vue psycho-sociologique la fonction de guide touristique maisen insistant surtout sur les divers fôles et statuts do ces cguides. Le processus d'interaction lui-meame est reste un sujet marginal. Voir COHEN, Erik (1985). The Tourist Gude: The Origins, Structures and Dynamics of A Roles, texted introduction au numéro spécial sur ce theme de The Annals of Tourism Research, vol, 12, no 1, pp. 6-29.

(14) Le tout premernuméro de Téros en février 1982 consacré a l'hoospitalité au Ouébec, avat tait ressortir notamment que le theme de l'hospitalite comme produit touristiquétaitd'autant plus utilise dans une région touristique que celle-gi recevait peu de touristes annumlement, et viceserga, Ne taudrait-1 pas reprendre une telle étude neuf ans plus tard?

(15) Voir divers articles sur ce sujet dans Teoros, wol 2. no 2, juin 1989; wegards neufs sur les attractonst touristiques du Quebec*, numeto coordonné par Marc Laplante.

(16) WATZLAVICK, Paulet autres/1979,, op. cit, p, 51 